\title{
Hypercalcemia associated with cosmetic injections: a systematic review
}

\section{Niranjan Tachamo', Anthony Donato², Bidhya Timilsina', Salik Nazir', Saroj Lohani', Rashmi Dhital' ${ }^{1}$ and Sijan Basnet ${ }^{1}$}

${ }^{1}$ Internal Medicine, Reading Hospital, Reading, Pennsylvania, USA and ${ }^{2}$ Internal Medicine, Sidney Kimmel Medical College at Thomas Jefferson University, Philadelphia, Pennsylvania, USA
Correspondence should be addressed to $\mathrm{N}$ Tachamo

Email

niranjantachamo@gmail.com

\begin{abstract}
Introduction: Cosmetic injections with silicone and polymethylmethacrylate are not FDA approved for augmentation of body parts such as breast, buttock or legs, but they have been widely used for decades. Cosmetic injections can cause foreign body granulomas and occasionally severe and life-threatening hypercalcemia. We aimed to systematically analyze the published literature on cosmetic injection-associated hypercalcemia.

Methods: We searched relevant articles on hypercalcemia associated with various cosmetic injections and extracted relevant data on demographics, cosmetic injections used, severity of hypercalcemia, management and outcomes. Results: We identified 23 eligible patients from 20 articles. Mean age was $49.83 \pm 14.70$ years with a female preponderance $(78.26 \%$ including transgender females). Silicone was most commonly used, followed by polymethylmethacrylate and paraffin oil $(43.48,30.43$, and $8.70 \%$ respectively). The buttock was the most common site followed by the breast $(69.57 \%$ and $39.13 \%$ respectively). Hypercalcemia developed at mean duration of $7.96 \pm 7.19$ years from the initial procedure. Mean ionized calcium at presentation was $2.19 \pm 0.61 \mathrm{mmol} / \mathrm{L}$ and mean corrected calcium at presentation was $3.43 \pm 0.31 \mathrm{mmol} / \mathrm{L}$. 1,25-Dihydroxyvitamin $\mathrm{D}\left(1,25(\mathrm{OH})_{2} \mathrm{D}\right.$ or calcitriol) was elevated while 25-hydroxyvitamin D (25(OH)D) and PTH were low in majority of cases. Hypercalcemia was managed conservatively with hydration, corticosteroids and bisphosphonates in majority of cases. Surgery was attempted in 2 cases but was unsuccessful. Renal failure was the most common complication $(82.35 \%$ cases $)$ and 2 patients died. Conclusion: Hypercalcemia from cosmetic injections can be severe and life threatening and can present years after the initial procedure. Cosmetic injection-associated granuloma should be considered a cause of hypercalcemia, especially in middle-aged females presenting with non-PTH-mediated, non-malignant hypercalcemia, which is often associated with elevated calcitriol; however, it should be noted that calcitriol level may be normal as well.
\end{abstract}

\section{Introduction}

Cosmetic surgeries are being increasingly performed worldwide. More than 11 million cosmetic surgeries were performed globally in 2013 with more than 1.4 million performed in US alone (1). Injectable fillers have gained popularity in the recent years because of their low cost and early recovery time in comparison to invasive surgery (2). Fillers including collagen, hyaluronic acid and
C) 2018 European Society of Endocrinology Printed in Great Britain polymethylmethacrylate (PMMA) are approved by the Food and Drug Administration (FDA) for augmentation of the face and back of hand, but not other anatomic sites. Silicone injection is not FDA approved for cosmetic augmentation of any tissues (3). Despite this lack of FDA approval, these fillers are frequently used for cosmetic augmentation of the breast and buttocks. Around 
313000 breast augmentations and around 12000 buttock augmentations/gluteoplasties including encapsulated fillers were performed in the US in 2013 alone (1).

Currently, there are more than 160 different injectable cosmetic products worldwide from more than 50 companies (2). Cosmetic injections can be associated with various complications, including injection site reactions, hypersensitivity and immune reactions, infections, migration of implants and foreign body granulomas (2). Unregulated use of these products outside of conventional medical facilities increases potential risk for harm and abuse. Cosmetic injection-induced foreign body granuloma is reported in only $0.02-1 \%$ cases and is thought to be related to the impurities, irregularity and volume of injected fillers (4).

In rare instances, these granulomas can lead to calcitriol-mediated hypercalcemia. The first case of cosmetic injection leading to granuloma-induced hypercalcemia for silicone was reported in 1984 (5), and the first case of PMMA-induced hypercalcemia was in 2014 (6). Overactivity of extra-renal CYP27B1 (also known as 1-alpha-hydroxylase) in activated macrophages in the granulomas is believed to lead to the pathological calcitriol production and subsequent hypercalcemia (7); however, calcitriol level may not always be elevated despite increased enzyme activity, especially if there is low 25-hydroxyvitamin $\mathrm{D}$ level to begin with. In those cases, the relative ratio of 25 -hydroxyvitamin D and 1,25-dihydroxyvitamin $\mathrm{D}$ might be more helpful than the absolute value of 1,25-dihydroxyvitamin D (8). Cosmetic injection-associated hypercalcemia often presents years after initial cosmetic injection and can be severe enough to cause renal failure and death. In this review, we sought to systematically review the literature to better understand the presentation, clinical course and outcomes of these patients with cosmetic injectionassociated hypercalcemia.

\section{Subjects and methods}

\section{Search strategy}

A systematic electronic search (Fig. 1) was conducted from MEDLINE (via Pubmed), EMBASE and ClinicalTrials. gov for case reports, case series, systematic reviews and clinical trials for synonyms of 'foreign body, silicone, polymethylmethacrylate, mineral oil, paraffin, dermal fillers' and 'hypercalcemia' from inception till December 26, 2016. This was done using highly sensitive search strategies in order to minimize the risk of excluding relevant studies (Supplementary File 1, see section on supplementary data given at the end of this article). These themes were combined using Boolean operator 'AND'. Search was limited to humans and English language articles. Search strategy was supplemented by hand-scanning of references of relevant publications and contacting authors for additional information.

\section{Inclusion criteria}

- Age $>18$ years.

- Hypercalcemia defined as serum calcium level $>2.6 \mathrm{mmol} / \mathrm{L}$ or ionized calcium $>1.4 \mathrm{mmol} / \mathrm{L}$.

- History of prior cosmetic injections with silicone, polymethylmethacrylate, paraffin oil, mineral oil, talc, etc.

- No other causes of hypercalcemia identified.

- Articles in English language.

\section{Exclusion criteria}

- No serum calcium values were reported.

\section{Data extraction and analysis}

Screening of articles for eligibility was performed by two authors (N T, B T). Conflicts during the screening process were resolved by the third author ( $\mathrm{S}$ ). Data extraction was performed by four authors (S L, S N, R D, S B). Data extracted included demographic variables, clinical presentation, clinical and laboratory investigations and patient outcomes (Supplementary File 2). Authors were contacted to elicit additional information if needed. Categorical variables were expressed as percentage and continuous variables as mean \pm S.D.

\section{Results}

We included 23 eligible patients from 20 articles (Table 1). Mean age of the patients was $49.83 \pm 14.70$ years. Eighteen of 23 patients (78.26\%) were females, including 6 transgender females. Six of 23 patients (26.09\%) were using the cosmetic injections for lipoatrophy associated with highly active antiretroviral therapy. The most commonly used cosmetic injections were silicone $(10 / 23$, $43.48 \%)$ followed by polymethylmethacrylate $(7 / 23$, $30.43 \%)$, paraffin oil (8.70\%) and mineral oil (8.70\%). The buttock was the most common site of cosmetic injection 


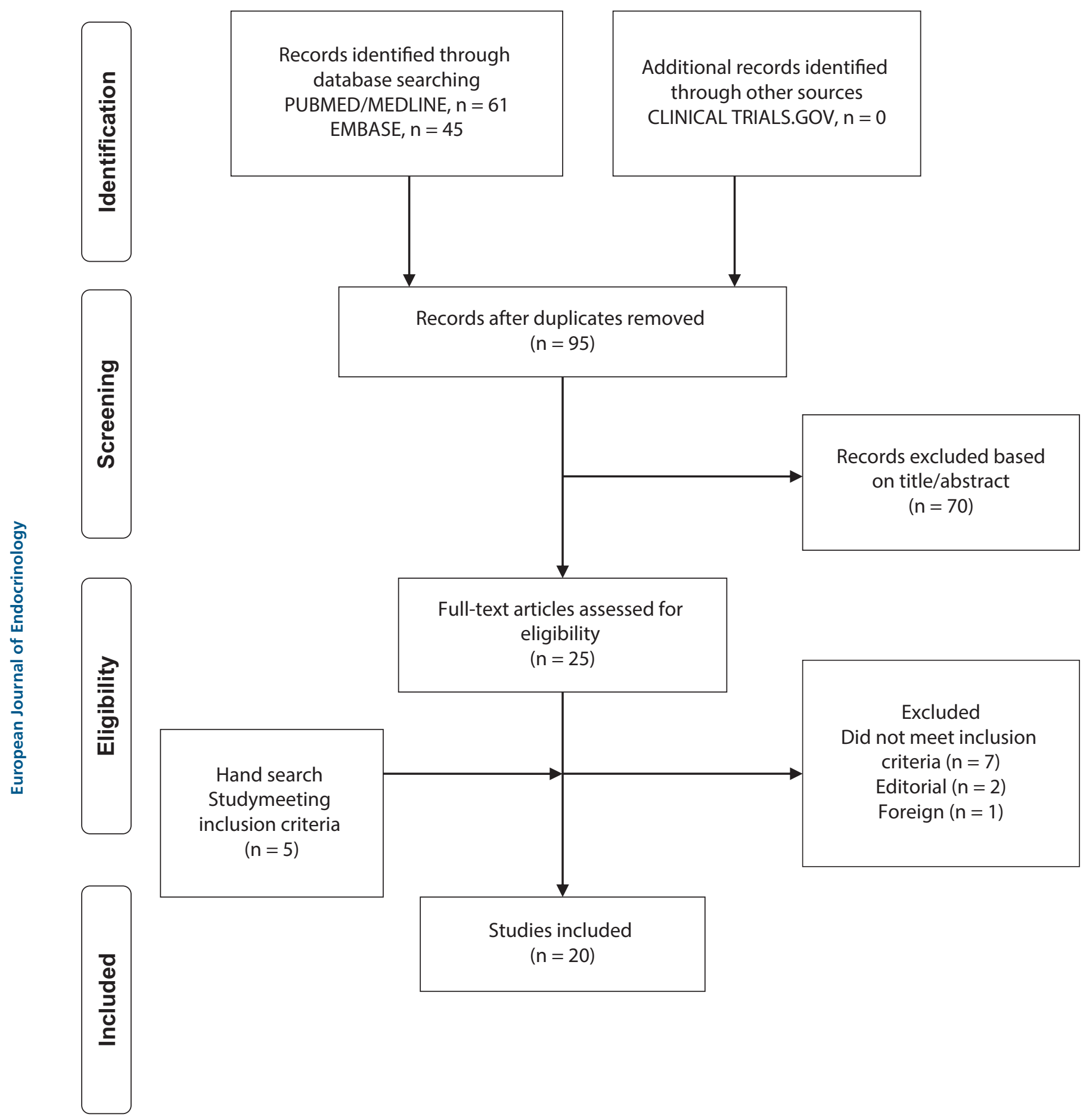

Figure 1

Flow chart describing systematic search and study selection process.

$(16 / 23,69.57 \%)$ followed by breast $(9 / 23,39.13 \%)$. The final target of the injection was intramuscular in nine of 23 cases (39.13\%), subcutaneous in eight of 23 cases $(34.78 \%)$ and both in 6 cases (26.09\%). The dose of foreign body injection was reported in only 2 cases, with average dose of $2.45 \mathrm{~L}$.

Hypercalcemia was diagnosed anywhere from few months to 28 years after initial cosmetic injection, with 
Table 1 Summary of results.

\begin{tabular}{|c|c|}
\hline \multicolumn{2}{|l|}{ Sex, $n(\%)$} \\
\hline Male & $5(21.74)$ \\
\hline Female & $12(52.17)$ \\
\hline Transgender females & $6(26.09)$ \\
\hline Age (years), mean \pm S.D. & $49.83 \pm 14.70$ \\
\hline \multicolumn{2}{|l|}{ Cosmetic injections used, $n(\%)$} \\
\hline Silicone & $10(43.47)$ \\
\hline Polymethylmethacrylate & $7(30.43)$ \\
\hline Both & $1(4.35)$ \\
\hline Paraffin oil & $2(8.70)$ \\
\hline Mineral oil & $2(8.70)$ \\
\hline Unknown & $1(4.35)$ \\
\hline \multicolumn{2}{|l|}{ Cosmetic injection sites, $n(\%)$} \\
\hline Buttock & $16(69.57)$ \\
\hline Breast & $9(39.13)$ \\
\hline Thigh & $4(17.39)$ \\
\hline Legs & $3(13.04)$ \\
\hline Arms & $3(13.04)$ \\
\hline Face & $3(13.04)$ \\
\hline Neck & $1(4.35)$ \\
\hline $\begin{array}{l}\text { Onset of hypercalcemia from initial injection } \\
\text { (years), mean } \pm \text { S.D. }\end{array}$ & $7.96 \pm 7.19$ \\
\hline Ionized calcium (mmol/L), mean \pm S.D. & $2.19 \pm 0.61$ \\
\hline $\begin{array}{l}\text { Total corrected calcium level (mmol/L), } \\
\text { mean } \pm \text { S.D. }\end{array}$ & $3.43 \pm 0.31$ \\
\hline $\begin{array}{l}\text { Presented with symptoms of hypercalcemia, } \\
n(\%)\end{array}$ & $17(73.91)$ \\
\hline Elevated calcitriol level, $n(\%)$ & $13(65)$ \\
\hline Suppressed PTH level, $n(\%)$ & $18(81.82)$ \\
\hline $\begin{array}{l}\text { Renal failure as complication of } \\
\text { hypercalcemia, } n(\%)\end{array}$ & $14(82.35)$ \\
\hline \multicolumn{2}{|l|}{ Medical management, $n(\%)$} \\
\hline Steroids & $20(86.96)$ \\
\hline Bisphosphonate & $12(52.17)$ \\
\hline Denosumab & $1(4.35)$ \\
\hline Calcitonin & $2(8.70)$ \\
\hline Ketoconazole & $1(4.35)$ \\
\hline Recurrent hypercalcemia, $n(\%)$ & $10(45.45)$ \\
\hline Death, $n(\%)$ & $2(8.70)$ \\
\hline
\end{tabular}

the mean duration being $7.96 \pm 7.19$ years. Ionized calcium was available in 5 cases, in which the mean ionized calcium was $2.19 \pm 0.61 \mathrm{mmol} / \mathrm{L}$. Corrected calcium could be calculated in 9 cases and the mean corrected calcium level at presentation was $3.43 \pm 0.31 \mathrm{mmol} / \mathrm{L}$. In 9 cases where corrected calcium could not be calculated, the average calcium level at presentation was $3.39 \pm 0.34 \mathrm{mmol} / \mathrm{L}$. 1,25-Dihydroxyvitamin $\mathrm{D}$ was normal or elevated in all the available cases where it was reported (normal in $7 / 20$, elevated in 13/20). 25-Hydroxyvitamin D was low or normal in all available cases (low in 13/19 and normal in 6/19). Parathyroid hormone (PTH) was suppressed or normal in the 22 cases reporting this value (suppressed in 18/22 and normal in 4/22). One patient developed hypercalcemia while on zoledronic acid for treatment of osteoporosis (9).
Presenting symptoms were nonspecific symptoms common to elevated serum calcium, including fatigue, weakness, dehydration, nausea, abdominal pain, constipation, polyuria, nocturia, cognitive decline and weight loss in $17 / 23$ patients (73.91\%). Only two of 23 patients $(8.70 \%)$ presented with symptoms at the injection site. Five cases reported utilizing Gallium scanning, and all of them showed increased uptake at sites of injection. Eight cases reported utilizing PET/CT scan, with 7 of those (87.50\%) showing increased metabolic activity at sites of injection. Biopsy of the involved skin or lymph node was performed in 20 cases, which revealed foreign body granulomatous reaction in 19/20 (95\%) cases and 1 case reporting an insufficient sample. Three cases mentioned doing immunochemical staining for expression of CYP27B1, with all 3 reporting strong expression of CYP27B1.

Hypercalcemia was managed conservatively with hydration and corticosteroids in 20 of 23 cases (86.96\%), was treated with bisphosphonates (pamidronate or zoledronic acid) in 12 of 23 cases (52.17\%) and denosumab was used in one case (4.35\%). Two cases were treated with calcitonin and one case with ketoconazole in addition to corticosteroids due to resistant hypercalcemia. Prednisone and methylprednisolone were the corticosteroids used (15 and 5 cases respectively). The most commonly used dose of prednisone was $30-40 \mathrm{mg} /$ day or $0.5 \mathrm{mg} / \mathrm{kg} / \mathrm{day}$ (range: $2.5-100 \mathrm{mg} /$ day) and tapered as calcium normalized. Dose of methylprednisolone was reported in one case only $(8 \mathrm{mg} /$ day). Calcium level normalized with prednisone in 9 cases in days to months. Pamidronate was the most commonly used bisphosphonate in 11 cases and was used most commonly with glucocorticoids; however, it was used alone in 1 case (10) and reported normalization of serum calcium with no recurrence in 1 year. One case had persistent hypercalcemia despite repeated steroids and calcitonin but resolved with ketoconazole $200 \mathrm{mg}$ every $8 \mathrm{~h}$ (6). Surgical resection of the granulomas was attempted only in 2 cases but was incomplete due to fragmented and encapsulated material in one case and extensive nature of the granulomatous infiltration in the other, and hence, medical management was continued (6). Recurrent hypercalcemia occurred in 10 of 22 cases $(45.45 \%)$. Renal failure was the most common complication, reported in $14 / 17$ cases $(82.35 \%)$ and was associated with 2 deaths. One patient who died had recurrent hypercalcemia with subsequent renal failure. Surgical reduction of the extensive paraffinoma burden in this patient was planned but could not be done due to renal failure and infectious complications (11). The other 
patient who died had recurrent hypercalcemia and UTI and died from UTI-related sepsis. She was also found to have anemia with hemoglobin of $7.6 \mathrm{~g} / \mathrm{dL}$ due to antral erosions from recurrent hypercalcemia (12).

\section{Discussion}

In this review, we noted many patients with an elevated 1,25-dihydroxyvitamin D (calcitriol) levels with suppressed PTH and low 25-hydroxyvitamin D levels in majority of the cases $(65,81.82,68.42 \%$ respectively), suggesting a calcitriol-mediated, PTH-independent mechanism of hypercalcemia, which is consistent with typical granulomatous reaction (13); however, calcitriol level may not always be elevated as identified in $35 \%$ patients in our study. The relative ratio of 25-hydroxyvitamin D and 1,25-dihydroxyvitamin D could not be calculated due to the heterogeneity of data. Biopsy of the granulomas were positive for CYP27B1 in the 3 cases that were tested $(7,14,15)$. Gallium scanning $(5,8,16,17,18)$ and PET/CT $(6,7,15,19,20,21)$ revealed increased uptake/metabolic activity in 12 of the 13 cases we identified where this imaging was employed.

We identified silicone as the most commonly used cosmetic injection, and breasts followed by buttocks as the most commonly injected sites, despite absence of FDA approval for this indication (3). We also identified a long delay in the presentation of hypercalcemia from the initial cosmetic injection, with the mean duration of 7.66 years.

Most of the cases included in our review were managed conservatively. Hydration along with corticosteroids (most commonly prednisone at dose of $30-40 \mathrm{mg} / \mathrm{day}$ or $0.5 \mathrm{mg} / \mathrm{kg} / \mathrm{day}$ ) with or without bisphosphonate (most commonly pamidronate) was the most commonly utilized treatment regimen and seemed effective to improve acute calcium levels in majority of cases. Surgical excision of granulomas was attempted only in 2 cases; however, it was incomplete as the granulomas were extensive. In the absence of proper guideline for management of cosmetic injectionassociated granulomas and hypercalcemia, both medical and surgical management have been attempted (13). Edwards et al. reported successful treatment of hypercalcemia refractory to medical management with surgical resection of the granuloma (22). However, resection is often not feasible or incomplete due to extensive and diffuse infiltrating nature of the granulomas, as identified in our review.
In our review, renal failure was the most common complication, occurring in $82.35 \%$ of reported cases and associated with 2 deaths. Recurrence of hypercalcemia following treatment occurred in $45.45 \%$ cases. As hypercalcemia in these cases is initiated by the granulomas, hypercalcemia would be expected to persist or recur unless the granuloma is resected. It is quite possible that hypercalcemia may still recur in the other cases where resolution of hypercalcemia was reported, given the foreign bodies causing the granuloma were not removed.

\section{Limitations}

Our study has some limitations. The quality and synthesis of data were dependent upon the individual cases, and there was heterogeneity among the data reported with multiple missing and incomplete data points (including corrected calcium, ionized calcium, Vitamin D3, Vitamin D2, PTH, patient outcomes and extended follow-up). Moreover, our study was limited to articles in English language, which may have caused us to exclude important non-English studies.

\section{Conclusion}

Hypercalcemia from cosmetic injections is rare but can be severe and life threatening. Patients, especially females, with non-PTH mediated, non-malignant hypercalcemia associated with elevated or normal calcitriol level should be asked about a history of prior cosmetic injections, as there can be a significant time delay in presentation. As the trend of cosmetic injections and cosmetic medical tourism continue to grow, more cases of foreign body granulomas with subsequent severe and life-threatening hypercalcemia will likely be reported. All these cases should be submitted to a registry with standardized reporting so that additional data may be available to better guide future guidelines, policies and interventions.

Supplementary data

This is linked to the online version of the paper at https://doi.org/10.1530/ EJE-17-0938.

Declaration of interest

The authors declare that there is no conflict of interest that could be perceived as prejudicing the impartiality of this study. 
Funding

This research did not receive any specific grant from any funding agency in the public, commercial or not-for-profit sector.

\section{Acknowledgements}

The authors are thankful to our librarians Alexandra Short (MSLS, AHIP) and Valerie Schaeffer (MSLS) for helping us find all the relevant articles.

\section{References}

1 http://www.isaps.org/Media/Default/global-statistics/2014\%20 ISAPS\%20Global\%20Stat\%20Results.pdf. Accessed on 7 October 2017.

2 Funt D \& Pavicic T. Dermal fillers in aesthetics: an overview of adverse events and treatment approaches. Clinical, Cosmetic and Investigational Dermatology 20136 295-316. (https://doi.org/10.2147/ CCID.S50546)

3 FDA. Soft tissue fillers (Dermal fillers). (available at: http:// www.fda.gov/MedicalDevices/ProductsandMedicalProcedures/ CosmeticDevices/WrinkleFillers/ucm2007470.htm\#Materials). Accessed on 13 February 2017.

4 Lemperle G, Gauthier-Hazan N, Wolters M, Eisemann-Klein M, Zimmermann U \& Duffy DM. Foreign body granulomas after all injectable dermal fillers: part 1. Possible causes. Plastic and Reconstructive Surgery 2009123 1842-1863. (https://doi.org/10.1097/ PRS.0b013e31818236d7)

5 Kozeny GA, Barbato AL, Bansal VK, Vertuno LL \& Hano JE. Hypercalcemia associated with silicone-induced granulomas. New England Journal of Medicine 1984311 1103-1105. (https://doi. org/10.1056/NEJM198410253111707)

6 Negri AL, Rosa Diez G, Del Valle E, Piulats E, Greloni G, Quevedo A, Varela F, Diehl M \& Bevione P. Hypercalcemia secondary to granulomatous disease caused by the injection of methacrylate: a case series. Clinical Cases in Mineral and Bone Metabolism 201411 $44-48$.

7 Hindi SM, Wang Y, Jones KD, Nussbaum JC, Chang Y, Masharani U, Bikle D, Shoback DM \& Hsiao EC. A case of hypercalcemia and overexpression of CYP27B1 in skeletal muscle lesions in a patient with HIV infection after cosmetic injections with polymethylmethacrylate (PMMA) for wasting. Calcified Tissue International 201597 634-639. (https://doi.org/10.1007/s00223-015-0048-8)

8 Visnyei K, Samuel M, Heacock L \& Cortes JA. Hypercalcemia in a male-to-female transgender patient after body contouring injections: a case report. Journal of Medical Case Reports 2014871.

9 Schanz J, Flux K, Kircher C, Tsioga M, Hartschuh W, Nawroth PP $\&$ Rudofsky G. Mirror, mirror on the wall: hypercalcemia as a consequence of modern cosmetic treatment with liquid silicone. Medical Science Monitor 201218 CS5-CS7.

10 Melnick S, Abaroa-Salvatierra A, Deshmukh M \& Patel A. Calcitriol mediated hypercalcaemia with silicone granulomas due to cosmetic injection. BMJ Case Reports 2016 2016. (https://doi.org/10.1136/bcr2016-217269)

11 El Muayed M, Costas AA \& Pick AJ. 1,25-Dihydroxyvitamin D-mediated hypercalcemia in oleogranulomatous mastitis (paraffinoma), ameliorated by glucocorticoid administration. Endocrine Practice 201016 102-106. (https://doi.org/10.4158/ EP09008.CRR)

12 Loke SC \& Leow MK. Calcinosis cutis with siliconomas complicated by hypercalcemia. Endocrine Practice 200511 341-345.

13 Granda ML \& Huang LE. Silicone injection-related granulomatous hypercalcemia. American Journal of the Medical Sciences 2017353 492-494. (https://doi.org/10.1016/j.amjms.2016.04.018)

14 Moraitis AG, Hewison M, Collins M, Anaya C \& Holick MF. Hypercalcemia associated with mineral oil-induced sclerosing paraffinomas. Endocrine Practice 201319 e50-e56. (https://doi. org/10.4158/EP12092.CR)

15 Gyldenløve M, Rørvig S, Skov L \& Hansen D. Severe hypercalcaemia, nephrocalcinosis, and multiple paraffinomas caused by paraffin oil injections in a young bodybuilder. Lancet 20143832098.

16 Agrawal N, Altiner S, Mezitis NHE \& Helbig S. Silicone-induced granuloma after injection for cosmetic purposes: a rare entity of calcitriol-mediated hypercalcemia. Case Reports in Medicine 2013 2013 807292. (https://doi.org/10.1155/2013/807292)

17 Camuzard O, Dumas P, Foissac R, Fernandez J, David S, Balaguer T, Chignon-Sicard B \& Dumontier C. Severe granulomatous reaction associated with hypercalcemia occurring after silicone soft tissue augmentation of the buttocks: a case report. Aesthetic Plastic Surgery 201438 95-99.

18 Khan O \& Sim JJ. Silicone-induced granulomas and renal failure. Dialysis and Transplantation 201039 254-259. (https://doi. org/10.1002/dat.20448)

19 Martin M, Anderson N \& Ranji S. An unforeseen reaction: a less common cause of hypercalcemia. Journal of General Internal Medicine 201530 S360.

20 Rados DV \& Furlanetto TW. An unexpected cause of severe and refractory PTH-independent hypercalcemia: case report and literature review. Archives of Endocrinology and Metabolism 201559 277-280. (https://doi.org/10.1590/2359-3997000000051)

21 Rey M, Lozano MP, Canalis M, Martínez MP \& Pozzo MJ. Hypercalcemia due to 1-25(OH)2D3 in a HIV patient. Bone 201149 1381.

22 Edwards BJ, Saraykar S, Sun M, Murphy WA, Lin P \& Gagel R. Resection of granulomatous tissue resolves silicone induced hypercalcemia. Bone Reports 20165 163-167. (https://doi. org/10.1016/j.bonr.2015.07.001)

Received 7 November 2017

Revised version received 1 February 2018

Accepted 16 February 2018 